\title{
Distributed Control Applications Within Sensor Networks
}

\author{
BRUNO SINOPOLI, STUDENT MEMBER, IEEE, COURTNEY SHARP, \\ LUCA SCHENATO, STUDENT MEMBER, IEEE, SHAWN SCHAFFERT, STUDENT MEMBER, IEEE,
} AND S. SHANKAR SASTRY, FELLOW, IEEE

\section{Invited Paper}

\begin{abstract}
Sensor networks are gaining a central role in the research community. This paper addresses some of the issues arising from the use of sensor networks in control applications. Classical control theory proves to be insufficient in modeling distributed control problems where issues of communication delay, jitter, and time synchronization between components are not negligible. After discussing our hardware and software platform and our target application, we review useful models of computation and then suggest a mixed model for design, analysis, and synthesis of control algorithms within sensor networks. We present a hierarchical model composed of continuous time-trigger components at the low level and discrete event-triggered components at the high level.
\end{abstract}

Keywords-Distributed control, distributed pursuit-evasion game (DPEG), embedded, Mica, mote, NesC, pursuit-evasion game (PEG), sensor network, TinyOS.

\section{INTRODUCTION}

Sensor networks (SNs) are gaining a role of importance in the research community. Embedded computers are well settled in our lives, in our houses, in our cars, and in our work environments.

Embedded systems, by definition, interact with the physical world. They are sensors, actuators, and controllers which are programmed to perform specified functions. As the range of applications grows, the need arises to network several embedded systems to perform incrementally complex tasks. The automotive domain is an excellent example. Here several embedded systems interact to provide a safe, comfortable driving experience.

Manuscript received December 13, 2002; revised March 14, 2003. This work was supported by the Defense Advanced Research Projects Agency (DARPA) under Grant F33615-01-C-1895.

The authors are with the Robotics and Intelligent Machines Laboratory, University of California, Berkeley, CA 94702 USA (e-mail: sinopoli@eecs.berkeley.edu; cssharp@eecs.berkeley.edu; lusche@eecs. berkeley.edu; sms@eecs.berkeley.edu; sastry@eecs.berkeley.edu).

Digital Object Identifier 10.1109/JPROC.2003.814926
Recent developments in MEMS technology have provided us with a wealth of cheap, customizable, embedded sensor systems capable of wireless communication among each other. The advantage of wireless SNs is enormous-deploying and maintaining a network of thousands of nodes is impractical considering the thousands of miles of wire that would be needed for the connections. Several hardware platforms are available, developed by both startups [1]-[3] and universities [4].

Applications in various fields of research are being developed. Interesting ongoing projects include extensive experimentation of structural response to earthquakes [5], habitat monitoring [6], and intelligent transportation systems [7]. Other important fields of applications include home and building automation and military applications. Self-configurable, ubiquitous, easy to deploy, secure, undetectable SNs are an ideal technology to employ in intelligence operations and war scenarios for detecting movements of enemy troops and artillery, and for monitoring and managing friendly resources.

The research community has quickly acknowledged the importance of large-scale ad hoc networks and has developed several services to support applications. Time services [8] provide the network with a globally consistent notion of time; localization services [9] allow computing nodes to acquire their coordinates relative to each other; routing services [10], [11] reliably deliver packets while dynamically adapting to the ever-changing network topology; and tracking services [12], [13] follow objects moving through the network.

System design and implementation on such a versatile platform introduces a series of issues. The longevity of these networks requires a stable software platform capable of self configuration, self upgrade, and adaptation to changing environmental conditions. Another set of issues arises when an SN is used for control applications. This is the thrust of this paper. Throughout our discussion, we will see several 
keys issues presenting themselves time and time again: location determination, time synchronization, reliable communication, power consumption management, cooperation and coordination, and security.

The goal of our research is to design robust controllers for distributed systems that violate typical control assumptions. Designed controllers will be evaluated on a distributed control application testbed. Among the wealth of available applications, we have selected a pursuit-evasion game (PEG) application. In our particular application, the SN is deployed in the environment where the game is played and cooperates with the pursuers' team.

This application includes many interesting research problems in the areas of tracking, control design, security, and robustness. For a PEG, the SN must be capable of multiple-vehicle tracking that can distinguish pursuers from evaders. Furthermore, the network needs to have a dynamic routing structure to deliver information to pursuers in minimal time. Since the game will be played in a distributed fashion, distributed sensing, control, and actuation need to be accounted for during controller design. To prevent the evader's team from intercepting sensitive information, the network must provide security features. Finally, since any one node of an SN can fail, control algorithms should show graceful performance degradation.

\section{PEGs}

The framework of PEGs captures fundamental features for modeling multiagents in cooperative robotics and has been an active area of research in the past decades. In this section, we give a brief overview of the research history on PEGs, describe the advantages of adding SNs to standard PEGs, and enumerate additional issues that arise when using SNs.

\section{A. PEG Overview}

PEGs are a mathematical abstraction arising from numerous situations which addresses the problem of controlling a swarm of autonomous agents in the pursuit of one or more evaders. Typical examples are search and rescue operations, surveillance, localization and tracking of moving parts in a warehouse, and search and capture missions. In some cases, the evaders are actively avoiding detection, as in capture missions, whereas in other cases their motion is approximately random, as in rescue operations.

Different versions of PEGs have been analyzed according to different frameworks and assumptions. Deterministic PEGs on finite graphs have been extensively studied [14], [15]. In these games, the playing field is abstracted to be a finite set of nodes, and the allowed motions for the pursuers and evaders are represented by edges connecting nodes. An evader is captured if both the evader and one of the pursuers occupy the same node. One of the most important problems arising from this framework is the computation of the search number, i.e., the smallest number of pursuers necessary to capture a single evader in a finite time, regardless of the escaping policy adopted by the evader. It has been shown that this problem is NP-hard [15], [16]. This approach is limited only to worst case motions of the evaders, and it is in general overly pessimistic. A great deal of research has focused on how to reduce a continuous space into a discrete number of regions, each to be mapped into a node of the graph, so that the game on the reduced graph is equivalent to the original game in the continuous space. For example, LaValle et al. proposed a method of decomposing the continuous space into a finite number of regions for known polygonal environments [17] and simply connected, smooth-curved, two-dimensional (2-D) environment [18].

Another active area of research deals with PEGs where the environment is unknown. In this framework, an additional map-learning phase is required to precede the pursuit phase. The map-learning phase is, by itself, time-consuming and computationally intensive even for simple 2-D rectilinear environments [19]. Moreover, inaccurate sensors complicate this process and a probabilistic approach is often required [20].

Finally, a recent approach to PEGs has dealt with combining map learning and pursuit into a single problem. This is done in a probabilistic framework to avoid the conservativeness inherent in worst case assumptions on the motion of the evader. A probabilistic framework also naturally takes into account inaccurate sensor readings, uncertain a priori map of terrain, and evaders motion policies [21], [22].

\section{B. SNs in PEGs}

The use of an SN can greatly improve the overall performance of a PEG. Pursuers have a relatively small detection range. They usually employ computer vision or ultrasonic sensors, providing only local observability over the area of interest. This constraint makes designing a cooperative pursuit algorithm harder because lack of complete observability only allows for suboptimal pursuit policies (see Fig. 1). Furthermore, a smart evader is difficult to catch unless appropriately detected.

Communication among pursuers may be difficult over a large area. Lack of communication, even partially, among pursuers is a major disruption for any pursuit policy. Because of the expense of unmanned vehicles, it is unrealistic to deploy a large number of them to continuously monitor a large region.

With SNs, complete visibility of the field and communication over a long radius is possible (see Fig. 2). Global pursuit policies can then be used to efficiently find the optimal solution regardless of the level of intelligence of the evader. Also, with an SN, the number of pursuers needed is likely a function exclusively of the number of evaders and not to the size of the field.

This distributed PEG (DPEG) scenario exposes a number of issues fundamental to any SN. Resolving these issues is complicated by the desire to make the solutions robust even in a dynamic ad hoc network.

Time-The notion of time presents two distinct problems. First, coordinating sensing and actuating in the physical world requires either a sense of global time or the ability to resolve different time measurements to a meaningful representation. Second, many existing design techniques 

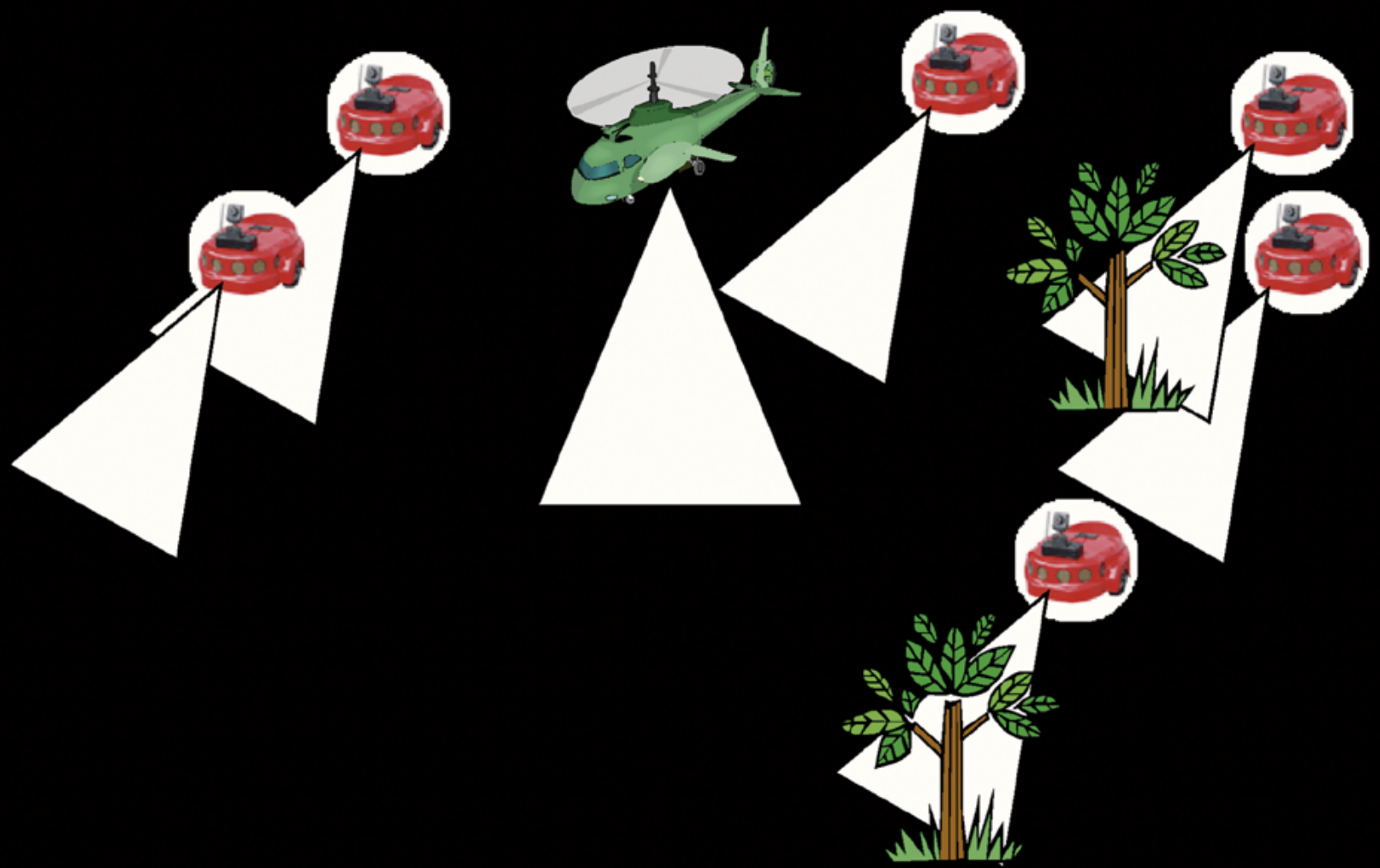

Fig. 1. PEG: what pursuers really see.

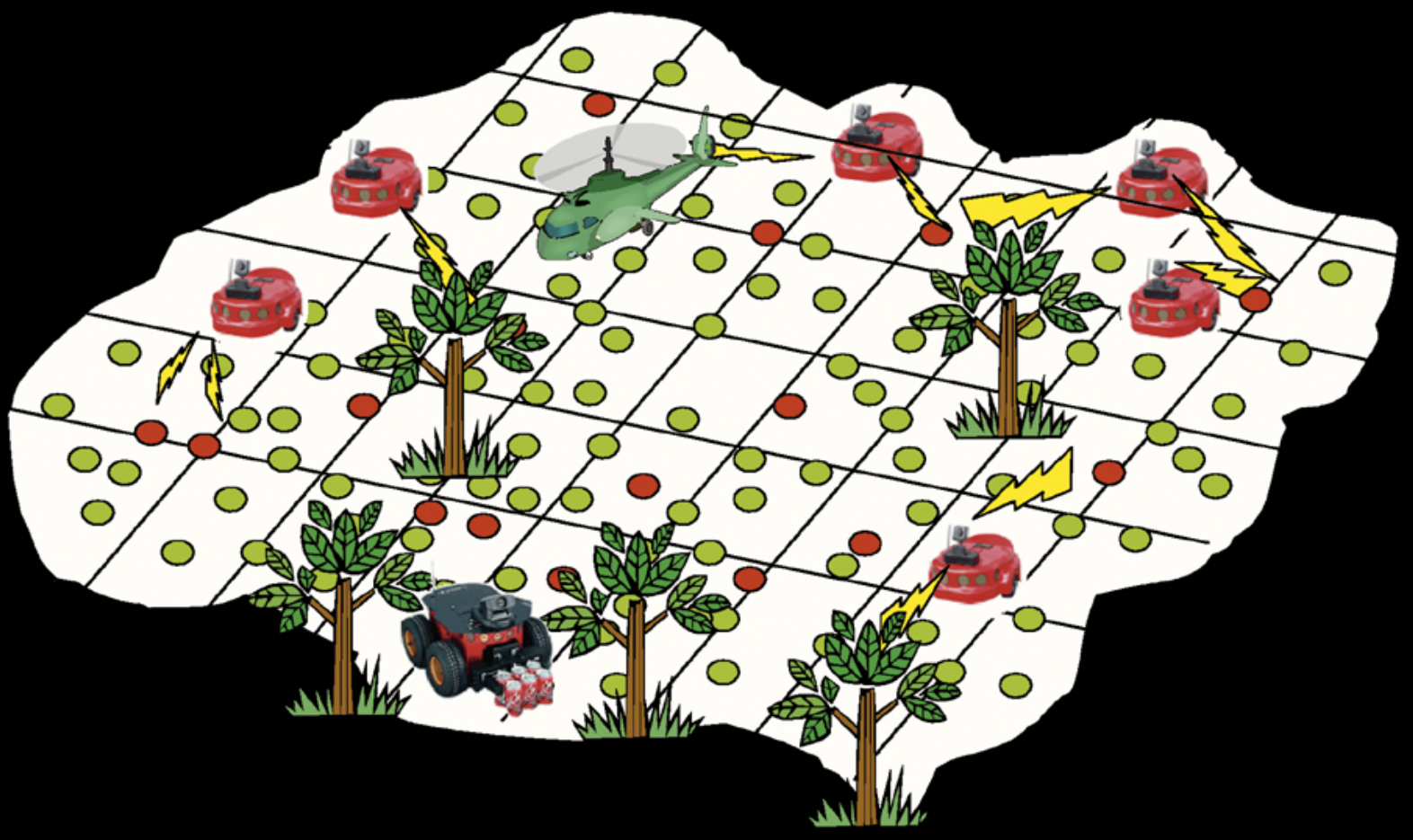

Fig. 2. PEG: SN increases visibility. 
assume that the computation of control and the processing of sensing and actuation occur within a negligible amount of time, thus requiring new design and analysis techniques for SNs.

Communication-It is expected that a network of motes will span a spatial area significantly greater than a single mote's maximum communication area. For a mote to send a message to another, distant mote, intermediate motes must be able to relay the message. Additionally, because motes can go offline without warning, the underlying communication protocol must be robust to network changes.

Location-Sensing and actuating events in the physical world must be paired with the relative or absolute location of the mote to be useful to control algorithms. That location must be assumed, provided, or deduced.

Cooperation-Tasks that require the combined effort of two or more motes, such as any form of distributed sensing or distributed computing, require protocols and structures that provide handshaking, coordination, and possibly hierarchy.

Power-Energy is a valued resource in an SN. Service and performance guarantees provided by an SN must be balanced against overall power consumption.

Security - To prevent numerous potential abuses of an $\mathrm{SN}$, a communication security layer must provide known guarantees for access control, message integrity, and confidentiality.

When developing control applications on an SN platform, we are particularly interested with issues related to time, communication, and location. We will focus on these issues throughout.

\section{DPEGs}

To start our DPEG scenario, the motes composing the SN are deployed onto the playing field in a sleep state. The mote $\mathrm{SN}$ then goes through an initialization and calibration stage for bootstrapping their provided services. The pursuers and evaders then enter the playing field and remain within the field for the duration of the game.

The SN provides a variety of services to both pursuers and other sensor motes: time synchronization, localization, moving entity (pursuer or evader) estimation, etc. For the purpose of the game, the sole goal of these services is to produce estimates on the positions, velocity, and identity of entities in the playing field. This information is time-stamped and routed to all pursuers in the playing field. The pursuers have onboard computation facilities comparable to a laptop computer. We may choose to have the pursuers communicate through a separate robust channel to coordinate to capture the evader when and if that channel is available.

When all evaders are captured (a capture occurs when a pursuer is "close enough" to it), the game ends. A base station is outside the playing area and provides logging and visualization services.

\section{IMPLEMENTATION}

Our implementations span hardware, software, and various application scenarios to explore and demonstrate distributed control via SNs. In the hardware section, we discuss our current embedded network devices. Then, in the software section, we review our new programming language, operating system (OS), and system service architecture. Finally, we survey our current and future testbeds for interacting and learning at the whole-system level.

\section{A. Hardware}

The hardware platform developed by the TinyOS group at Berkeley consists of numerous, small, extendable embedded network devices. Each device has limited power, computation, and storage resources-significantly limited when compared to modern desktop computer systems. The goal of each hardware platform is to provide computation, sensing, actuation, and communication resources embedded in miniature packaging. By making the conscious design decision to significantly limit the resources available per mote, we leave the door open for reaching the goal of dust-sized devices.

The current platforms are designed to be both modular and flexible, providing ease in retargeting motes to new and unanticipated applications while allowing for significant code reuse. Fig. 3 shows the evolution of the base computation modules. In particular, the most recent transition from the Rene2/Dot to Mica (see Fig. 4) gave at least a fourfold increase in program memory, RAM, and radio transmission rate. All motes have otherwise had some form of a 4-MHz, 8-b Atmel microcontroller and an RFM TR1000 radio.

Add-on boards for the motes may be designed for generalpurpose sensing or targeted toward a particular application. For instance, the weather sensing board has humidity, barometric pressure, infrared, temperature, and light sensors, and is used for experiments on Great Duck Island [23] in Maine. And a motor/servo and whisker/accelerometer board were developed for COTS-BOTS [24] (see Fig. 5) for controlling off-the-shelf miniature cars. We also have various generalpurpose sensor boards that have some combination of photodiodes, temperature sensors, magnetometers, accelerometers, microphones, and sounders.

The overall modularity of these devices comes at the cost of size. A device targeted at large-scale deployment can do away with the add-on connector and supporting circuitry. The resulting space savings in the current platforms easily allows for a final form-factor with diameter smaller than a quarter.

All together, the hardware platforms have been sufficient to meet the needs of both research and experimentation.

\section{B. System Services}

We build our embedded software with NesC [25], a new, open-source programming language developed at Berkeley. NesC extends the standard $C$ language with semantics and syntax for component-based architectures. Component behaviors are described with bidirectional interfaces that either provide commands or require the dependent to handle events. Components are statically wired together to form a whole program or system, which, when compiled with a wholeprogram compiler, allows for greater optimizations and efficiency. Whole programs also match well with formal analysis tools for verifying system functionality. 


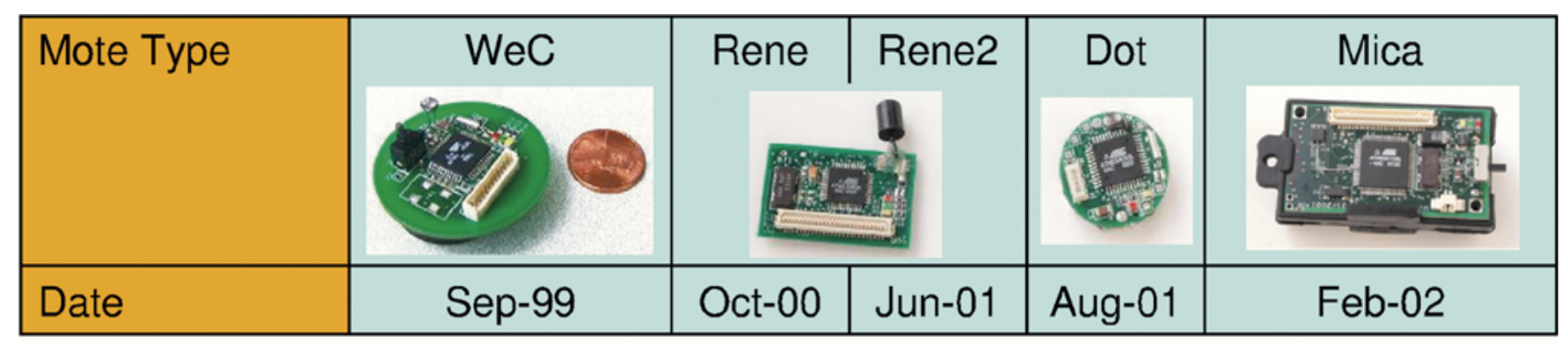

Microcontroller (4MHz)

\begin{tabular}{|l|c|c|c|}
\hline Type & AT90LS8535 & ATMega163 & ATMega103/128 \\
\hline Prog.Mem.(KB) & 8 & 16 & 128 \\
\hline RAM (KB) & 0.5 & 1 & 4 \\
\hline
\end{tabular}

Communication

\begin{tabular}{|l|c|c|}
\hline Radio & \multicolumn{2}{|c|}{ RFM TR1000 } \\
\hline Rate (Kbps) & 10 & $10 / 40$ \\
\hline ModulationType & OOK & OOK/ASK \\
\hline
\end{tabular}

Fig. 3. Evolution of motes from the Berkeley TinyOS group.

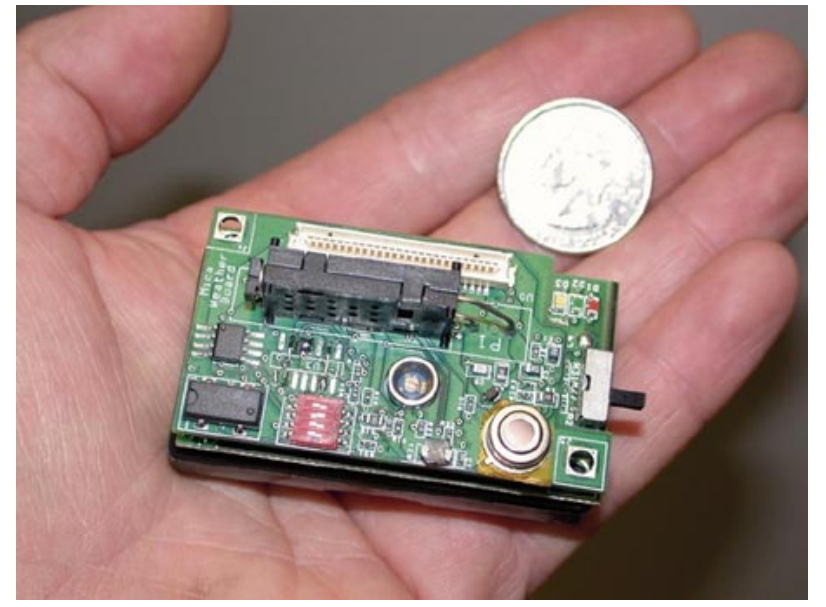

Fig. 4. Mica mote $\left[2^{\prime \prime} \times 1^{\prime \prime} \times(3 / 4)^{\prime \prime}\right]$ with attached weather board module.

Berkeley's open-source embedded OS, TinyOS [26], provides basic system services, such as communication and simple process scheduling, and access to hardware components, such as sensors and actuators. It is specifically designed for extremely resource-limited devices that have only a few kilobytes of memory. TinyOS is written in NesC using a component-based architecture with layered access to hardware resources, which provides robustness, flexibility, and extensibility.

Using NesC and TinyOS as building blocks, we have been working with a number of other groups on the NEST project funded by the Defense Advanced Research Projects Agency (DARPA) to develop a coherent architecture of system services to help solve fundamental $\mathrm{SN}$ stumbling issues. The

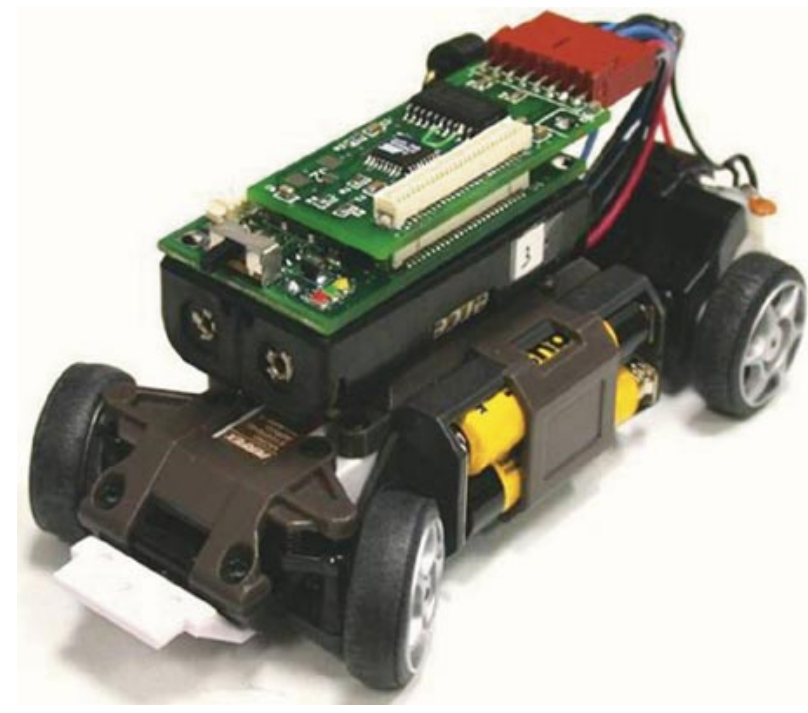

Fig. 5. COTS-BOTS developed by S. Bergbreiter and K. Pister.

crucial services we have currently identified are estimation, grouping, localization, power management, routing, service coordination, and time synchronization. We feel that these components will facilitate a large set of rich and adaptive applications.

To address time issues within an $\mathrm{SN}$, we propose a time synchronization application programming interface (API) that supports two time management protocols: a global Network Time Protocol (NTP)-like synchronization protocol, and a local time protocol with the means to transform time readings between individual motes. It is expected that NTP-like global synchronization will offer lower precision 
time measurements, but otherwise provide an immediately available global time on the mote. Local transformations between individual mote "time zones" have the advantages of higher precision between pairs of motes, being able to back-calculate synchronized times for past events, and guarantees monotonicity in local time by not directly modifying the local clock [8]. Various applications can have vastly different time synchronization requirements, and we feel these two methodologies together can more adequately serve a broad set of applications.

To address communication issues within an SN, we propose a general routing framework that supports a number of routing methodologies. First, because SNs primarily sense and interact with phenomena in the physical world, routing to geographic regions is expected to be the common case. Second, to assist in routing packets around physical obstacles, routing based on geographic direction is expected to be useful. Third, the more obvious case of routing to symbolic network identifiers is reserved for dynamically routing to physically moving destinations within the network. Finally, the general case of constraint-based routing provides means to route based on arbitrary criteria, such as power level, sensor values, and so on.

To resolve the physical location of motes in an SN [9], we propose a top-to-bottom localization framework. A localization service requires a broad set of coordination and processing stages between motes: coordinated sensors and actuators, group data management, and computation. Separating localization into a number of distinct components that work together allows for an amount of heterogeneity in the $\mathrm{SN}$ that may be necessary given the limited resources of the motes.

The issue of coordination between motes needs to be addressed. We propose both application-specific grouping algorithms and general-purpose grouping services. A group management service must provide means to send and receive data from a group, the ability to join and leave a group, and leader election. For tracking a moving evader in a PEG scenario, decisions to join and leave groups can be tied to sensor readings. This simplifies the handshaking and decision process, allowing for overall lower overhead. There are concerns that these services in the general case impose significant overhead on an SN.

Issues of power management are on the agenda but are currently unaddressed in the architecture. Issues of security are being solved at the OS level, providing transparent authentication, encryption, and concealment.

Our methodology for creating an infrastructure for these services is to first specify a set of prototypes that define abstract programming interfaces for classes of components and services. Developers then create components that instantiate an algorithm using one or more prototypes. Some components may behave as services whose execution and behavior are managed by a central coordinator. Finally, interaction between components must be formalized by specifying protocols and types. Fig. 6 illustrates this methodology in a sample architecture that shows the interactions and protocols between components, services, a service coordinator, sensors,

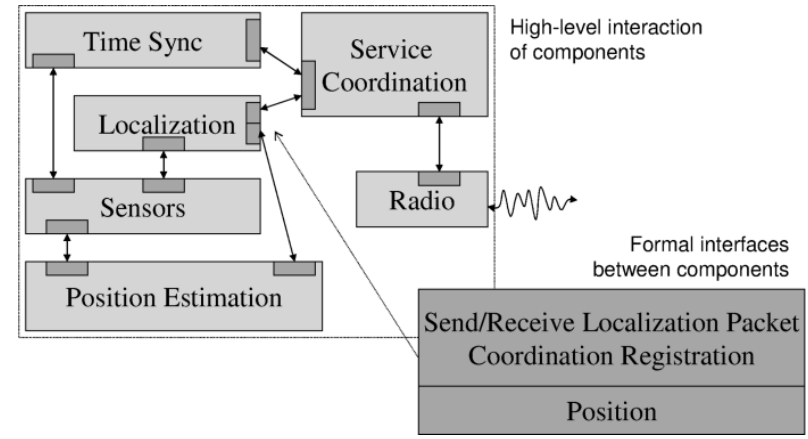

Fig. 6. Sample component architecture demonstrating the design methodology.

and radio channel. Fig. 7 further shows the relationship between these services, components, TinyOS, and a DPEG application layer.

\section{Testbeds}

Our current experimental platform is functional but limited when compared to the scope of a full DPEG scenario. It is the result of a focused effort to produce a solution for a set of particular goals rather than to provide a general framework. To that end, it exists more as a proof that a highly constrained DPEG solution is achievable and that NesC and TinyOS provide a suitable platform for development.

Fig. 8 shows the setup for that platform. A human remotely controls a miniature car, and the $\mathrm{SN}$ remotely controls a pan-tilt-zoom camera to track the car. Because we have not yet integrated a self-localization service on the motes, the SN is a uniform grid of 25 motes, where each mote presumes its location given its network address. Each mote shares its location with its local neighborhood, which is necessary both for position estimation and geographic-based routing. When a mote detects change in its local magnetic field, it broadcasts its readings to its local neighbors and records similar broadcasts from other, nearby motes. In this way, local behaviors are expected; currently, we are not attempting to aggregate readings from the entire network to produce a single, global estimate. The mote with the highest reading is implicitly elected the leader, who calculates a position estimate from its cached neighborhood readings. That estimate is sent via reliable geographic-based multihop routing to a base station mote, which relays it to a camera mote. The camera mote performs the actuation necessary to point toward the estimated location.

What we would like to do is to use the SN software architecture to implement this scenario in a more versatile, general framework. We are looking forward to a more complete, outdoor PEG scenario, shown in Fig. 9. Beyond that scenario, we look forward to expanding our understanding of whole-system behavior through formalism and parameterization of distributed SNs.

\section{Methodology}

Our initial DPEG implementation has provided valuable insight into the pressing issues that a control design method- 

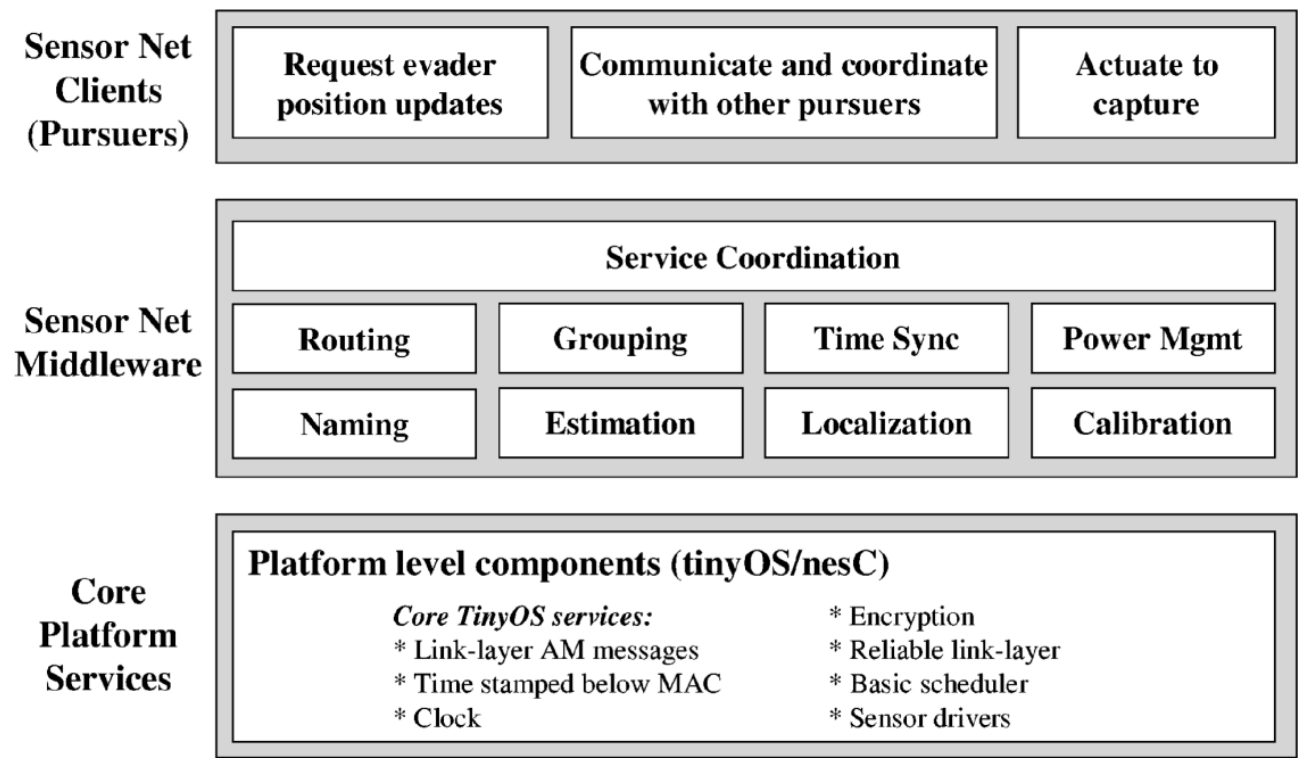

Fig. 7. Relationship between proposed services and components.

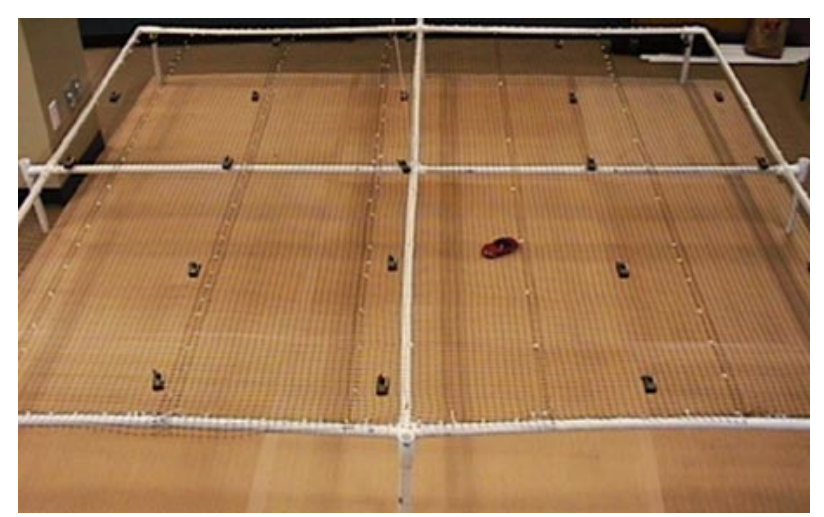

Fig. 8. Indoor sensor-based tracking testbed.

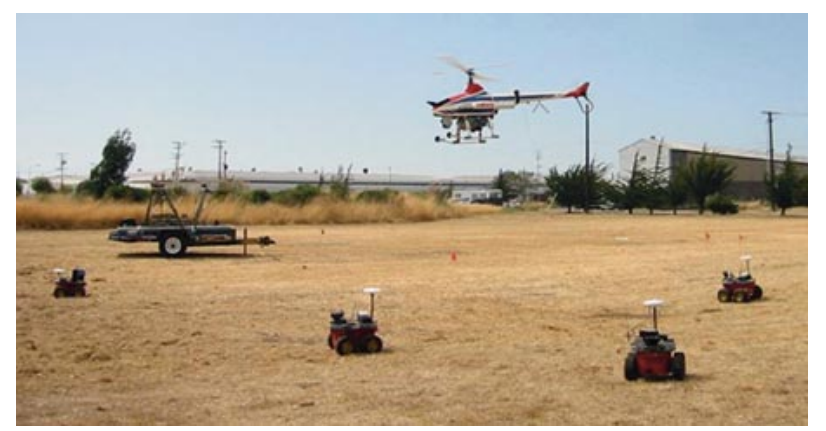

Fig. 9. Outdoor DPEG testbed.

ology must address, and we will use these ideas to inform our proposed design methodology. In this section, we will first review existing design approaches that address the issues of scalability and distribution. During this discussion, we will be interested in extracting the essence of existing algorithms while abstracting away the particular choice of model. Following this, models of computation (MOCs) will be explored that are useful for describing such systems. Finally, we will discuss our proposed design methodology that will be applied to the next DPEG implementation.

\section{A. Scalability and Distributed Control}

Distributed control systems are an integral part of our world and have been studied in many different contexts, ranging from biology to artificial intelligence to control systems. Naturally occurring distributed systems such as ants searching for food, bacteria foraging, and the flight formations of some birds have been well studied by biologists and are beginning to receive more attention from other communities interested in distributed algorithms. Indeed, the artificial intelligence community has considered such systems in more abstract terms for several years. Additionally, the continuous time control community has addressed many of the features that distinguish distributed control systems from classical centralized control systems.

Nature provides us with several good examples of distributed control in action. For example, schooling in fish [27] and cooperation in insect societies [28] exhibit complex collective patterns arising from rather simple individual behavior. These social behaviors have been argued to improve food search, predator avoidance, and colony survival for the species as a whole rather than for the individual. Some researchers have been turning to such examples to gain insight into these naturally optimized distributed algorithms. Investigating bacteria foraging of E. coli, Passino [29] has developed a distributed optimization algorithm. The algorithm models how $E$. coli bacteria move in a solution as they collectively search for nutrients and avoid toxins to reach an optimal state where the collection of bacteria is satisfied with their surroundings.

The artificial intelligence community has addressed such systems under the title of distributed agents for several years [30]. Some researchers in this community have developed approaches such as free market systems [31] that mimic our own trade system. In this architecture, each agent, which could be a robot with a specialized ability, bids on a particular task based on its cost function which combines the robot's reward and effort. It is even possible for robots to be- 


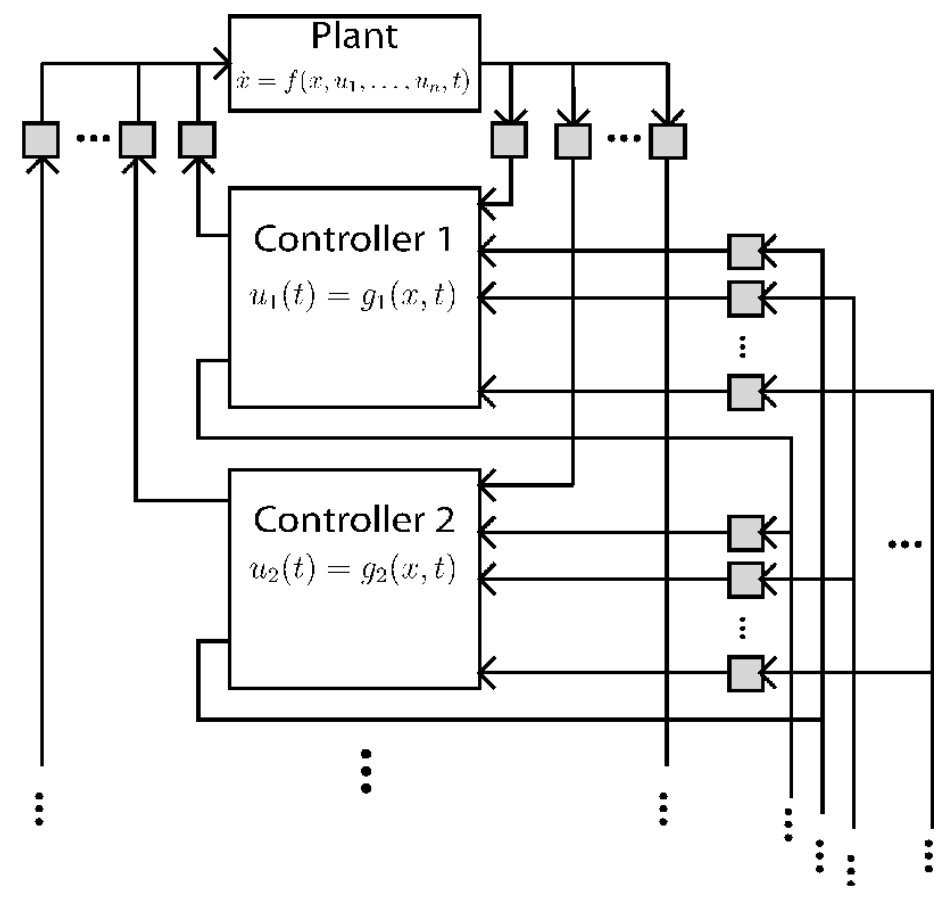

Fig. 10. Section of a distributed continuous control MOC with sensing, actuation, and communication jitter. Shaded blocks represent a time delay.

come leaders who bid on tasks and then subcontract the task out to several other robots.

The continuous control community has wrestled with distributed systems for many years in the realm of process control and has independently addressed many of the caveats of distributed systems, such as jitter compensation and scheduling. Martí et al. [32] have identified the types of jitter that can occur in distributed systems and investigated compensation techniques. Their method first analyzes whether online or offline compensation is needed. If online compensation is feasible, then the parameters of the control law are dynamically updated according to the next time the controller will be executed. Other researchers have reformulated the typical scheduling problem as a dynamic system so that the techniques of control theory may be applied [33]. In [34], a centralized scheduling rule is replaced with local instantiations of integral controllers that are shown to drive the state to a viable solution.

\section{B. Models of Computation}

The impossibility of characterizing these systems within the classical control framework raises the need to select one or more MOCs in order to accurately analyze distributed control problems in SNs. Our hope would be that such a combination captures the continuously changing dynamics of the environment, the distribution of resources, and the discrete nature of the hardware. To address this issue more specifically, we investigate several common MOCs, including discrete events, continuous dynamical systems, discrete-time dynamical systems, hybrid automata, synchronous reactive languages, and dataflow models. For each of these, we consider its advantages and its drawbacks with respect to control applications within SNs.
Continuous time dynamical systems [35], [36] are a wellstudied formal model. Key properties such as stability and reachability can be deducted using available analytical and numerical methods. Controllers can be designed to meet desired specifications. Additionally, they are familiar to the control community and, hence, preferred for control applications. However, for distributed control applications in SNs, this theory is not able to capture communication delays, time skew between clocks, or discrete decision making. Since all the variables are continuous, it is difficult to model such discrete phenomena. Additionally, controllers must be implemented on microprocessors, and control must be piecewise constant.

To describe the controller's piecewise constant nature, we turn to discrete time dynamical systems [35], [36]. However, we are again limited to characterizing systems without mode changes. Additionally, this MOC assumes periodic activation of the controller with instantaneous computation of the control law which is not preserved by the underlying platform. This model does not directly address sensing and actuation jitter, but it can be taken into account by augmenting with time delays between the plant and the controller. This approach assumes that the control law is computed synchronously on each node every $T$ seconds, but different sensing and actuation jitters are allowed for each node. This model is useful when we assume that the process scheduler running on each node can ensure synchronous operation. Additionally, the system can be modified to distribute control computation across nodes with state communication between them, as shown in Fig. 10.

The multimodal nature of such systems can be described by a hybrid automaton [37]. These systems nicely account for both the "continuous flow" and discrete jumps of such 
systems. Note that "continuous flow," or just flow, in a hybrid automaton may be modeled by either differential equations or difference equations. They allow the system to evolve according to the flow with occasional discrete transitions. Additionally, with each discrete transition, the equations governing the flow are allowed to change. Difference equations allow such a model to capture the piecewise constant nature of the controller. Mode changes can then be characterized by the discrete dynamics, where all the discrete properties of our application must be encoded. The discrete dynamics are similar to finite-state machines in that encoding many discrete variables leads to a discrete state explosion problem and quickly becomes unmanageable for SNs.

To consider MOCs more appealing for algorithms, we can consider discrete event systems [38]. Such a model works well for mode changes or task scheduling and characterizes the hardware platform nicely, as well. It also allows for the system to be event-triggered, which is often the case in SNs. However, it does not support continuous variables, and given the discrete nature of variables we again run up against a state explosion problem when modeling a large number of nodes. Finally, such systems generally do not correlate time steps of the model with real time.

Dataflow [39] MOCs are intended to describe data transformations. In particular, they are useful for characterizing several communicating processes. However, this paradigm is awkward for control, since it generally considers the relationship between sequences of inputs and sequences of outputs, rather than the evolution of the output for each input signal in turn. In general, when composing several dataflow models in a feedback loop, the result may not be deterministic [40].

Another set of common modeling paradigms are synchronous reactive languages, such as Signal [41], Lustre [42], and Esterel [43]. These languages support a broad range of formal verification tools to aid in debugging. Additionally, it is possible to generate code for the platform directly from the synchronous reactive language. However, we again find that there is no relation between time steps of the language and real time. Furthermore, synchronous reactive systems presume the existence of a global clock and that time steps-and, hence, the computation of fixed points-happen instantaneously. This MOC is not appropriate because it is not congruent with the event triggered nature of SNs. Finally, such a model can be counterintuitive, since it searches for a fixed point at every step.

\section{Design Approaches}

In the previous two sections, we described different approaches to address scalability and synchronus/asynchronous systems. Our approach to scalability for DPEGs will rely heavily on distributed processing of sensors readings in order to get good estimates of positions and velocities of both evaders and pursuers. The control of each pursuer dynamics is performed within the pursuer itself based on network readings, but higher level coordination will be distributed between all the pursuers to maximize robustness to adversarial attack. In order to address the issues arising from the fact that DPEGs include synchronous and asynchronous dynamics, several ad hoc solutions are available. To compensate for nonuniform time delays, one approach is to buffer the incoming data for a certain amount of time such that most of the data has arrived. With this approach, the problem has been reduced to the classical control problem of driving a system with a fixed time delay. However, this result comes at the price of suboptimal performance. As for missing data, the most common solutions are either using the most recent data regardless of its exact time of arrival, or estimating the most probable measurement that is consistent with previous measurements and the dynamics of the system.

Some issues related to the event-triggered nature of distributed control have been addressed by the hybrid system control community. Here, the idea is to develop a formalism that combines the best of control theory and state machine theory [44]-[46]. Although few analytical results are available today, this rather intuitive and promising approach is an active area of research.

Time synchronization research for SNs has been intense, yielding promising results [8]. In our model, we confidently assume that sensor readings come with an accurate time stamp. Also, we assume that sensors know their location in space. A localization service ensures that the nodes in a deployed network can compute their location relative to each other [9]. With these two assumptions, we use the standard control formalism with SNs. A choice of a model is critical to the design of controllers for such systems. In dealing with complex applications such as DPEGs, control must be exercised at several levels, and a hierarchical system seems to be the natural modeling choice. A graphical representation is shown in Fig. 11.

At the low level, the continuous time dynamics of the system need to be captured. Since the implemented controllers are digital, the model is discretized to yield a discrete time control system. At this level, the system is time based, in the sense that time triggers each transition. At each time step, an observation, generated from a sensor reading, needs to be provided to the controller, which will in turn produce an input to the dynamics of the system via an actuator. In standard control problems, the sensors are physically attached to the plant; therefore, it is assured to receive a sensor reading at each time step. In the case of SNs, the sensing is distributed. This means that it may take some time for the observation to reach its destination, since packets over the network are subject to delay and loss. Additionally, the control law needs some information about the plant to compute the next input, which will heavily rely on state estimation, prediction, and smoothing. In the absence of an observation, we will make use of the model alone to provide state estimation for control. In this way, late packets can be used to improve current estimate. Several methods can be used for estimation, from Kalman to particle filtering. A graphical representation of the low-level controller is shown in Fig. 12. 


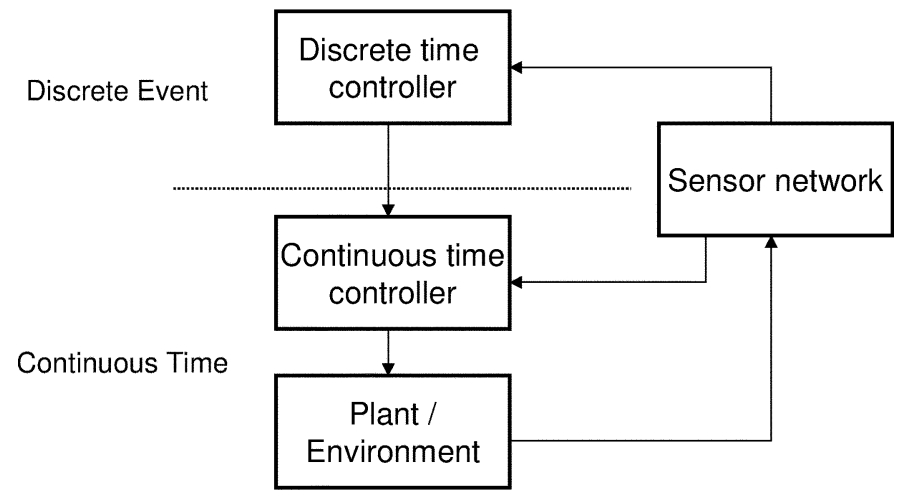

Fig. 11. A hierarchical system representation.

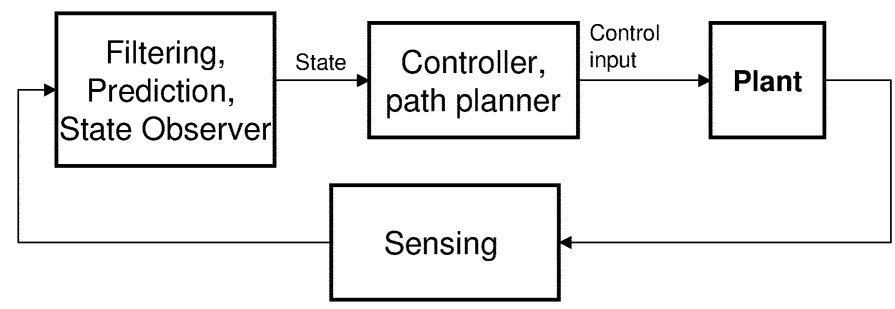

Fig. 12. Low-level controller.

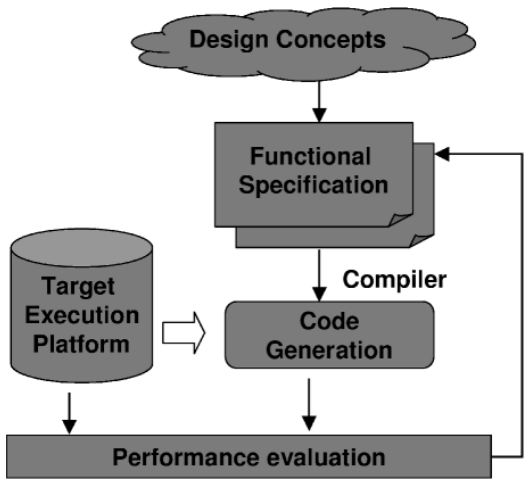

Fig. 13. The proposed design methodology.

At the higher level, the system is event based. In this domain, the control reacts to one or more events, sequences of which are called behaviors. Events are detected by the SN and transmitted to a discrete controller that generates the appropriate reaction. Each reaction is then transmitted to the lower level by changing the control objective to agree with the new specifications. Once again, events occur in an asynchronous fashion, making formal analysis difficult. To work with such events, we implement the system using a synchronous reactive language, where behaviors can be verified and mapped to our asynchronous platform, making sure the verified properties are preserved. The problem of mapping behaviors from different domains has been tackled in several different ways. We follow the approach of Benveniste [47] by designing controllers in a synchronous fashion, verifying the behavior, and then desynchronizing the algorithm to be implemented on the asynchronous target architecture. The advantage of this approach also includes the possibility of automatically generating embedded code directly from a high-level specification language, thus enormously speeding up the development phase. A graphical representation of the design flow is shown in Fig. 13.

\section{CONCLUSION}

In this paper, we presented an overview of research activities dealing with distributed control in SNs. We introduced $\mathrm{SNs}$ and related research issues. We then presented our hardware and software platforms while proposing an open architecture to help develop rich distributed applications. We presented an overview of the theoretical issues facing researchers interested in using SNs for distributed control applications. We identified key properties that cause classical control theory to fail. We suggested a general approach to control design using a hierarchical model composed of continuous time-triggered components at the low level and discrete event-triggered components at the high level. In the future, we will focus on implementation, verification, and testing of our methodologies in distributed control systems on our proposed DPEG testbed.

\section{ACKNOWLEDGMENT}

The authors would like to thank the TinyOS team for providing a great hardware and software platform. Additionally, the authors would like to thank the entire NEST team at Berkeley and Intel Research-Berkeley. Finally, the authors would like to thank the following individuals: S. Bergbreiter, E. Brewer, D. Culler, D. Gay, J. Hill, B. Hohlt, C. Karlof, P. Levis, S. Madden, K. Pister, J. Polastre, N. Sastry, R. Szewczyk, R. von Behren, D. Wagner, M. Welsh, K. Whitehouse, and A. Woo. 


\section{REFERENCES}

[1] Crossbow. [Online] Available: http://www.xbow.com/

[2] Millennial. [Online] Available: http://www.millennial.net

[3] Sensoria. [Online] Available: http://www.sensoria.com

[4] B. Warneke, M. Last, and K. S. J. Pister, "Smart Dust: Communicating with a cubic-millimeter computer," IEEE Computer, vol. 34, pp. 44-51, Jan. 2001.

[5] Smart buildings admit their faults. Lab Notes [Online] Available: http://www.coe.berkeley.edu/labnotes/1101smartbuildings.html

[6] A. Mainwaring, J. Polastre, R. Szewczyk, D. Culler, and J. Anderson, "Wireless sensor networks for habitat monitoring," presented at the 1st ACM Int. Workshop Wireless Sensor Networks and Applications, Atlanta, GA, 2002.

[7] A. N. Knaian, "A wireless sensor network for smart roadbeds and intelligent transportation systems," Ph.D. dissertation, Mass. Inst. Technol., Cambridge, 1999.

[8] J. Elson, L. Girod, and D. Estrin, "Fine-grained network time synchronization using reference broadcasts," presented at the 5th Symp. Operating Systems Design and Implementation (OSDI), Boston, MA, 2002.

[9] S. Klemmer, S. Waterson, and K. Whitehouse. (2000, Dec.) Toward a location-based context-aware sensor infrastructure. [Online] Available: http://guir.berkeley.edu/projects/location/Location.pdf

[10] D. Estrin, R. Govindan, J. S. Heidemann, and S. Kumar, "Next century challenges: scalable coordination in sensor networks," in Proc. 5th Annu. ACM/IEEE Int. Conf. Mobile Computing and Networking, 1999, pp. 263-270.

[11] E. M. Royer and C.-K. Toh, "A review of current routing protocols for ad-hoc mobile wireless networks," IEEE Pers. Commun., vol. 6 , pp. 46-55, Apr. 1999

[12] F. Zhao, J. Shin, and J. Reich, "Information-driven dynamic sensor collaboration,” IEEE Signal Processing Mag., vol. 19, pp. 61-72, Mar. 2002.

[13] L. Guibas, "Sensing, tracking and reasoning with relations," IEEE Signal Processing Mag., vol. 19, no. 2, pp. 73-85, Mar. 2002.

[14] T. Parsons, "Pursuit-evasion in a graph," in Lecture Notes in Mathmatics, Theory and Application of Graphs, Y. Alani and D. Lick, Eds: Springer-Verlag, 1976, vol. 642, pp. 426-441.

[15] M. Megiddo, S. Hakimi, M. Garey, S. Johnson, and C. Papadimitriou, "The complexity of searching a graph," J. ACM, vol. 35, no. 2, pp. 18-44, 1988.

[16] A. S. Lapaugh, "Recontamination does not help to search a graph," J. ACM, vol. 40, no. 2, pp. 224-245, Apr. 1993.

[17] S. LaValle, D. Lin, L. Guibas, J. Latombe, and R. Motwani, "Finding an unpredictable target in a workspace with obstacles," in IEEE Int. Conf. Robotics and Automation, vol. 1, 1997, pp. 737-742.

[18] S. LaValle and J. Hinrichsen, "Visibility-based pursuit-evasion: the case of curved environments," in IEEE Int. Conf. Robotics and Automation, vol. 3, 1999, pp. 1677-1682.

[19] X. Deng, T. Kameda, and C. Papadimitriou, "How to learn an unknown environment I: the rectilinear case," J. $A C M$, vol. 45 , no. 2 , pp. 215-245, 1998.

[20] S. Thrun, W. Burgard, and D. Fox, "A probabilistic approach to concurrent mapping and localization for mobile robots," Mach. Learn./Autonomous Robots (joint issue), vol. 31, no. 5, pp. 1-25, 1998.

[21] J. Hespanha, H. J. Kim, and S. Sastry, "Multiple-agent probabilistic pursuit-evasion games," in Proc. 38th Conf. Decision and Control, 1999, pp. 2432-2437.

[22] J. Kim, R. Vidal, H. Shim, O. Shakernia, and S. Sastry, "A hierarchical approach to probabilistic pursuit evasion games with unmanned ground and aerial vehicles," Proc. 40th IEEE Conf. Decision and Control, pp. 634-639, 2001.

[23] Great Duck Island. [Online] Available: http://www.greatduckisland. net/

[24] S. Bergbreiter and K. Pister. COTS-BOTS. [Online] Available: http://www-bsac.eecs.berkeley.edu/ sbergbre/CotsBots/cotsbots. html

[25] NesC. [Online] Available: http://nescc.sourceforge.net/

[26] TinyOS. [Online] Available: http://webs.cs.berkeley.edu/tos/

[27] J. Parrish, S. Viscido, and D. Grunbaum, "Self-organized fish schools: an examination of emergent properties," Biol. Bull., vol. 202, pp. 296-305, June 2002.

[28] J. Deneubourg, A. Lioni, and C. Detrain, "Dynamics of aggregation and emergence of cooperation," Biol. Bull., vol. 202, pp. 262-267, June 2002
[29] K. M. Passino, "Biomimicry of bacterial foraging for distributed optimization and control," IEEE Control Syst. Mag., vol. 22, pp. 52-67, June 2002.

[30] G. Weiss, Ed., Multiagent Systems: A Modern Approach to Distributed Artificial Intelligence, 2nd ed. Cambridge, MA: MIT Press, 2000.

[31] M. Dias and A. Stentz, "Opportunistic optimization for market-based multirobot control," presented at the IEEE/RSJ Int. Conf. Intelligent Robots and Systems, Lausanne, Switzerland, 2002.

[32] P. Martí, G. Fohler, K. Ramamritham, and J. M. Fuertes, "Jitter compensation in real-time control systems," in Real-Time Systems Symp. London, UK, 2001.

[33] M. Caramanis and A. Sharifnia, "Near optimal manufacturing flow control design," Int. J. Flexible Manuf. Syst., vol. 3, no. 3-4, pp. 321-336, June 1991.

[34] V. V. Prabhu and N. A. Duffie, "Nonlinear dynamics in distributed arrival time control," IEEE Trans. Control Syst. Technol., vol. 7, pp. 724-730, Nov. 1999.

[35] F. M. Callier and C. A. Desoer, Eds., Linear System Theory, 1st ed. New York: Springer-Verlag, 1991.

[36] C.-T. Chen, Ed., Linear System Theory and Design, 3rd ed. London, UK: Oxford Univ. Press, 1999.

[37] C. Tomlin, J. Lygeros, and S. Sastry, "Computing controllers for nonlinear hybrid systems," in Lecture Notes in Computer Science, Hybrid Systems: Computation and Control, F. W. Vaandrager and J. H. van Schuppen, Eds. Heidelberg, Germany: Springer-Verlag, 1999, vol. 1569 , pp. 238-255.

[38] E. A. Lee, "Modeling concurrent real-time processes using discrete events," Annals Softw. Eng. (Special Volume on Real-Time Software Engineering), vol. 7, pp. 25-45, 1999.

[39] W. B. Ackerman, "Data flow languages," IEEE Computer, vol. 15, pp. 15-25, Feb. 1982

[40] E. A. Lee, "A Denotational semantics for dataflow with firing," Electron. Res. Lab., Univ. of Cal., Berkeley, Tech. Rep. No. UCB/ERL M97/3, 1997.

[41] A. Benveniste and P. L. Guernic, "Hybrid dynamical systems theory and the signal language," IEEE Trans. Automat. Contr., vol. 35, pp. 525-546, May 1990

[42] P. Caspi, D. Pilaud, N. Halbwachs, and J. A. Plaice, "LUSTRE: A declarative language for programming synchronous systems," in Conf. Rec. 14th Annu. ACM Symp. Principles of Programming Languages, 1987, pp. 178-188.

[43] G. Berry and G. Gonthier, "The Esterel synchronous programming language: design, semantics, implementation," Sci. Comput. Program., vol. 19, no. 2, pp. 87-152, Nov. 1992.

[44] R. Alur and T. Henzinger, "Modularity for timed and hybrid systems," in Lecture Notes in Computer Science, CONCUR97: Concurrency Theory, 8th International Conference. Berlin, Germany: Springer-Verlag, 1997, vol. 1243.

[45] M. S. Branicky, V. S. Borker, and S. K. Mitter, "A unified framework for hybrid control: model and optimal control theory," IEEE Trans. Automat. Contr., vol. 43, pp. 31-45, Jan. 1998.

[46] J. Lygeros, C. Tomlin, and S. Sastry, "Controllers for reachability specifications for hybrid systems," Automatica, vol. 35, no. 3, pp. 349-370, Mar. 1999.

[47] A. Benveniste, "Some synchronization issues when designing embedded systems from components," in Lecture Notes in Computer Science, Proceedings of the First International Workshop on Embedded Software. Berlin, Germany: Springer-Verlag, 2001, vol. 2211.

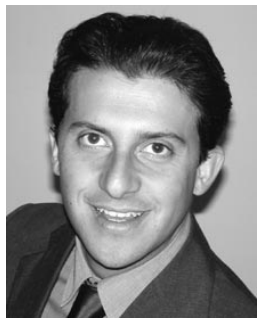

Bruno Sinopoli (Student Member, IEEE) received the Laurea degree in electrical engineering from the University of Padova, Padova, Italy in 1998. He is currently working toward the Ph.D. degree in electrical engineering from the University of California, Berkeley, under the supervision of Prof. S. Sastry.

His research interests include sensor networks, design of embedded systems from components, distributed control, and hybrid systems. 


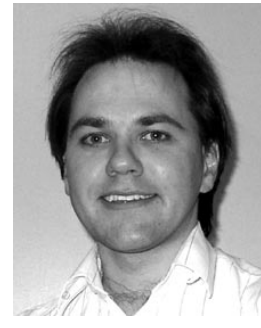

and computer vision.

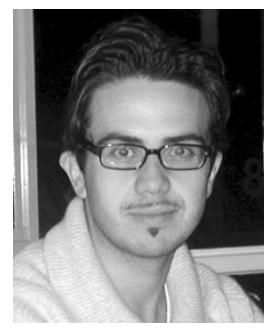

Luca Schenato (Student Member, IEEE) was born in Treviso, Italy, in 1974. He received the Dr. Eng. degree in electrical engineering from the University of Padova, Padova, Italy, in 1999. $\mathrm{He}$ is currently working toward the Ph.D. degree from the Department of Electrical Engineering, University of California, Berkeley.

His research interests include modeling of biological networks, insect locomotion, millirobotics, and avionics.

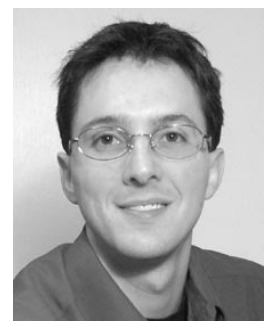

Shawn Schaffert (Student Member, IEEE) received the B.S. degree in electrical engineering from the University of Nebraska, Lincoln, in 1998, and the M.S. degree in electrical engineering from the University of California, Berkeley, in 2001. He is currently working toward the Ph.D. degree at the University of California, Berkeley, under the supervision of his research adviser, Prof. S. Sastry.

In 2001, he was an Intern at Xerox PARC, Palo Alto, CA, investigating the complexity of constrained optimization problems. His research interests include embedded systems, distributed control, hybrid systems, and robust control in sensor networks.

Mr. Schaffert is a member of Tau Beta Pi and Eta Kappa Nu.

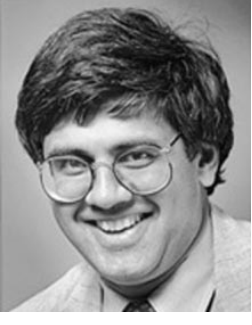

S. Shankar Sastry (Fellow, IEEE) received the M.S. degree (honoris causa) from Harvard University, Cambridge, MA, in 1994, and the Ph.D. degree from the University of California, Berkeley, in 1981.

From 1980 to 1982, he was an Assistant Professor at Massachusetts Institute of Technology, Cambridge. In 2000, he was Director of the Information Technology Office at the Defense Advanced Research Projects Agency, Arlington, VA. He is currently the NEC Distinguished Professor of Electrical Engineering and Computer Sciences and Bioengineering and the Chairman of the Department of Electrical Engineering and Computer Sciences, University of California, Berkeley. His research interests are embedded and autonomous software, computer vision, computation in novel substrates such as DNA, nonlinear and adaptive control, robotic telesurgery, control of hybrid systems, embedded systems, sensor networks, and biological motor control.

Dr. Sastry was elected into the National Academy of Engineering in 2001 "for pioneering contributions to the design of hybrid and embedded systems." He has served as Associate Editor for IEEE TRANSACTIONS ON Automatic Control, IEEE Control Systems Magazine, and IEEE TRANSACTIONS ON CIRCUITS AND SYSTEMS. 